\title{
¿Influye la modalidad de infusión de nutrición enteral en la tolerancia gastrointestinal del paciente en estado crítico? Revisión narrativa de la literatura
}

\author{
¿Is there an association between gastrointestinal intolerance and enteral \\ feeding in the critically ill patient? A narrative review of the literature \\ A modalidade de infusão da nutrição enteral influência a tolerância gastrointestinal do \\ paciente em estado crítico? - revisão narrativa da literatura
}

Iván Armando Osuna Padilla ${ }^{1,2}$, Francisco Gerardo Yanowsky Escatell ${ }^{3}$, Nadia Carolina Rodríguez-Moguel².

Recibido: 7 de septiembre de 2021. Aceptado para publicación: 25 de septiembre de 2021.

Primero en línea: 27 de septiembre 2021.

https://doi.org/10.35454/rncm.v4n4.345

\section{Resumen}

Introducción: la nutrición enteral (NE) es la vía de elección para administrar soporte nutricional al paciente en estado crítico. Aunque los beneficios inmunológicos y clínicos de la NE se encuentran bien documentados, no está exenta de complicaciones; y la modalidad de infusión es un factor que podría contribuir a intolerancia gastrointestinal (GI). Esta última se relaciona con la interrupción de la infusión, lo que conlleva una menor adecuación nutrimental e impacta negativamente en el estado nutricional y los desenlaces clínicos. Esta revisión tiene como objetivo evaluar las diferencias en la tolerancia Gl entre la modalidad de infusión intermitente/bolos y la infusión continua en individuos en estado crítico que reciben NE.

Método: se realizó una revisión de la literatura que comparó las diferencias en la tolerancia Gl entre ambas modalidades de infusión en el período de enero de 2010 a diciembre de 2020.

Resultados: se identificaron 27 estudios, de los cuales 4 fueron incluidos por cumplir con el criterio de comparar la modalidad de infusión de NE y tolerancia GI. Existe evidencia de una menor incidencia de intolerancia con una modalidad de infusión continua; sin embargo, esto varía

\section{Summary}

Introduction: Enteral nutrition (EN) is the preferred route to provide nutritional support in critically ill patients. Immunological and clinical benefits of EN are well documented, even if it is not exempt of complications, the modality used for its infusion being a factor that could contribute to gastrointestinal (GI) intolerance. $\mathrm{Gl}$ intolerance results in discontinuation of the infusion, leading to caloric debt and a negative impact on nutritional status and clinical outcomes. The objective of this study was to evaluate the differences in GI tolerance between intermittent and continuous enteral feeding modalities in critically ill individuals.

Methods: For this purpose, a literature review was carried out to compare the differences in $\mathrm{Gl}$ tolerance between both infusion modalities in the period from January 2010 to December 2020.

Results: Twenty-seven studies were identified. Four studies met the criterion of comparing EN infusion modality and GI tolerance incidence and were included. There is evidence of a lower incidence of EN intolerance with continuous enteral feeding; however, this varies according to the evaluated $\mathrm{Gl}$ tolerance parameter. According to the literature, studies have

\section{Resumo}

Introdução: a nutrição enteral (NE) é a via de escolha para administrar suporte nutricional ao paciente em estado crítico. Embora os benefícios imunológicos e clínicos da NE estejam bem documentados, não é isenta de complicações; sendo a modalidade de infusão um fator que poderia contribuir para a intolerância gastrointestinal (GI). A intolerância Gl está relacionada à interrupção da infusão que leva a uma menor adequação nutricional, impactando negativamente o estado nutricional e os desfechos clínicos. Esta revisão tem como objetivo avaliar as diferenças na tolerância Gl entre a modalidade de infusão intermitente / bolus e a infusão contínua em indivíduos em estado crítico que recebem NE.

Método: para isso, foi realizada uma revisão da literatura que comparou as diferenças de tolerância Gl entre as duas modalidades de infusão no período de janeiro de 2010 a dezembro de 2020.

Resultados: foram identificados 27 estudos, dos quais 4 foram incluídos por cumprirem com o critério de comparação da modalidade de infusão de NE e a tolerância GI. Existe evidência de menor incidência de intolerância com a modalidade de infusão contínua, porém, isso varia de 
según el parámetro de tolerancia Gl evaluado. Algunos estudios reportan mayor incidencia de residuo gástrico elevado y diarrea en el grupo con NE intermitente, mientras que otros han documentado mayor incidencia de estreñimiento con la NE continua.

Conclusión: se requieren ensayos clínicos aleatorizados que evalúen la incidencia de intolerancia Gl después de comparar las modalidades de infusión.

Palabras clave: nutrición enteral, soporte nutricional, paciente en estado crítico, diarrea, vómito, estreñimiento. observed a higher incidence of gastric residual volume and diarrhea in the intermittent feeding group, whereas continuous feeding has been associated with higher incidence of constipation.

Conclusion: Randomized clinical trials are required to assess the incidence of $\mathrm{Gl}$ intolerance after comparing infusion modalities.

Keywords: enteral nutrition, critically ill patient, nutritional support, diarrhea, vomiting, constipation. acordo com o parâmetro de tolerância $\mathrm{Gl}$ avaliado. Alguns estudos relatam uma maior incidência de resíduo gástrico elevado e diarreia no grupo com NE intermitente, enquanto outros documentaram uma maior incidência de constipação com a NE contínua.

Conclusão: ensaios clínicos randomizados são necessários para avaliar a incidência de intolerância Gl após a comparação das modalidades de infusão.

Palavras-chave: nutrição enteral, suporte nutricional, paciente em estado crítico, diarreia, vômito, constipação.
Departamento de áreas críticas, Instituto Nacional de Enfermedades Respiratorias "Ismael Cosío Villegas". Ciudad de México, México.

2 Centro de Investigación en Enfermedades Infecciosas, Instituto Nacional de Enfermedades Respiratorias "Ismael Cosío Villegas". Ciudad de México, México.

\section{INTRODUCCIÓN}

La nutrición enteral (NE) representa la vía de acceso a priorizar en el paciente en estado crítico, ya que el favorecimiento de la motilidad se ha asociado con el mantenimiento de la integridad de la barrera intestinal, el equilibrio en la composición de la microbiota y la respuesta inmunitaria mediada por el tejido linfoide asociado con el intestino; de este modo se contribuye con la modulación de la respuesta inflamatoria ${ }^{(1,2)}$.

Los beneficios de la NE están bien descritos; sin embargo, existe controversia con respecto a la modalidad de administración de la fórmula. La infusión continua se refiere a la provisión de fórmula a una tasa constante durante las 24 horas del día, mientras que la infusión cíclica consiste en la administración durante menos de 24 horas (por ejemplo, infusión nocturna durante 12 horas), ambas modalidades administradas mediante una bomba de infusión. La infusión en bolos e intermitente se refiere a la provisión de un volumen determinado 4 a 6 veces al día, cuya duración fluctúa entre 3 y 10 minutos por gravedad en bolos y 20-60 minutos en la modalidad intermitente; esta última a través de una bomba de infusión (Figura 1). En la literatura, el término de "infusión intermitente" se utiliza para referirse a las modalidades no continuas ${ }^{(3,4)}$.

La intolerancia gastrointestinal (GI) es un problema frecuente en el paciente en estado crítico, con una prevalencia que oscila entre un $23 \%$ y $26 \%$ según los resul-
Departamento de Medicina Interna, Hospital Civil de Guadalajara Dr. Juan I. Menchaca. Departamento de Ciencias de la Salud - Enfermedad como Proceso Individual, Centro Universitario de Tonalá, Universidad de Guadalajara Guadalajara, Jalisco, México.

*Correspondencia: Francisco Gerardo Yanowsky Escatell. fyanowsky@hotmail.com

tados de un estudio multicéntrico ${ }^{(5)}$. Esta problemática de origen multifactorial (respuesta inflamatoria, alteraciones hormonales y del sistema nervioso autónomo, desequilibrio hidroelectrolítico, descontrol glucémico, farmacoterapia) se ha asociado con una menor adecuación nutrimental y mayor deuda calórica. Se ha descrito que quienes presentan intolerancia reciben entre $11 \%$ y $15 \%$ menor aporte calórico con respecto a quienes no presentan intolerancia, lo que impacta negativamente en el estado nutricional y los desenlaces clínicos de este grupo de pacientes ${ }^{(6)}$.

Adicional a los factores previamente mencionados, la modalidad de infusión de la NE puede incrementar la intolerancia GI. Se ha documentado una disminución en los episodios de diarrea con el uso de infusión continua (riesgo relativo [RR]: 0,42; intervalo de confianza [IC] $95 \%$ : 0,19-0,91, $p=0,03$ ); sin embargo, no está del todo descrito el rol de la modalidad de alimentación en otras alteraciones gastrointestinales ${ }^{(7)}$. Diversas sociedades científicas internacionales han propuesto recomendaciones para la optimización de la terapia médica nutricional en los pacientes en estado crítico y sugieren la utilización tanto de infusión continua como intermitente/bolos para la prescripción de $\mathrm{NE}$; esto considerando la tolerancia esperada a la NE y el tipo de acceso de alimentación a utilizar ${ }^{(7-9)}$.

La presente revisión narrativa de la literatura surge debido a que la evidencia actual no ha abordado el impacto de las modalidades de infusión sobre los 


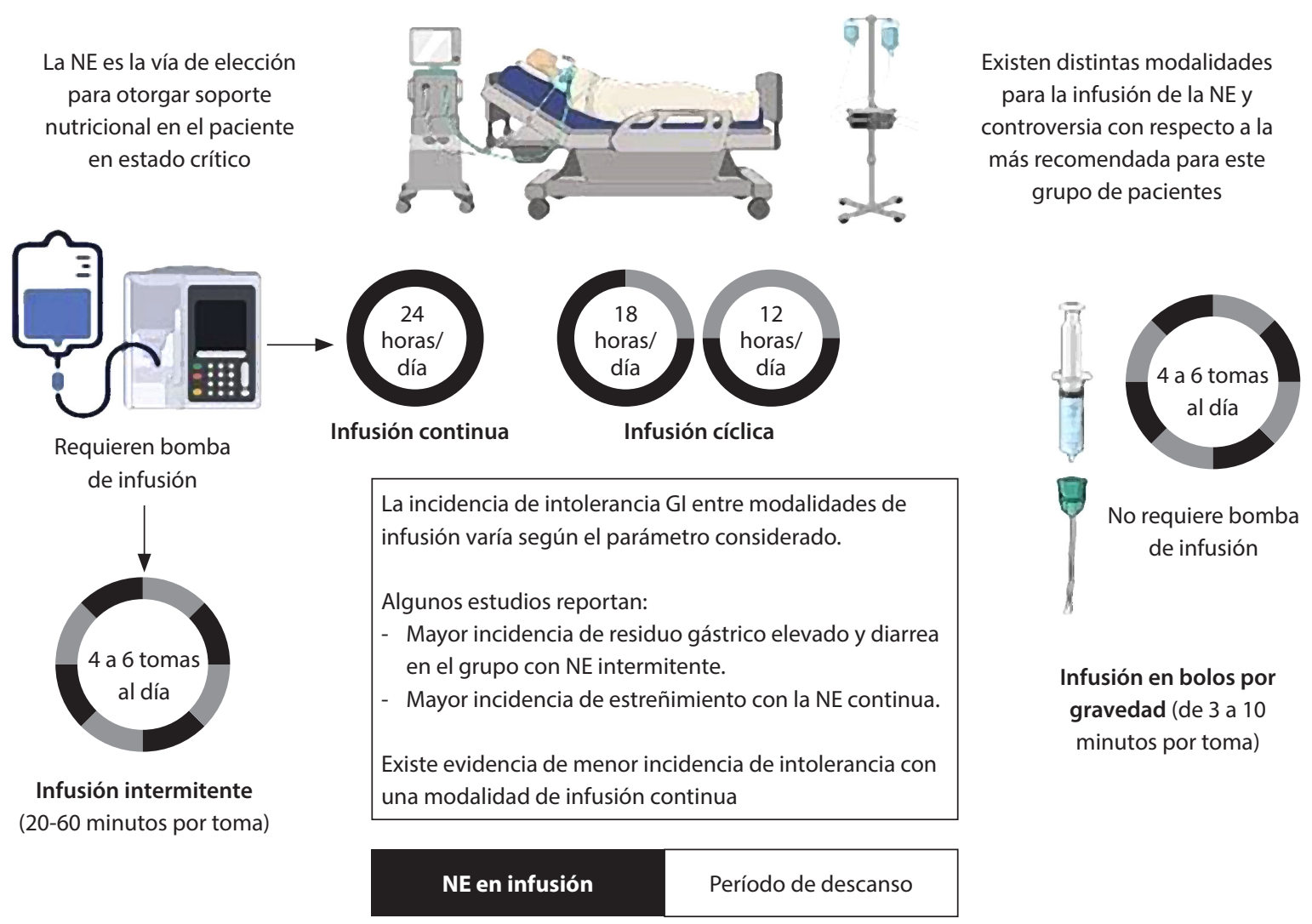

Figura 1. Modalidades para la infusión de la NE. La administración de una fórmula enteral en el paciente críticamente enfermo puede realizarse de manera continua o fraccionada en tomas en un período de 24 horas, para ello pueden utilizarse dispositivos electrónicos que controlen la cantidad a infundir. Existe evidencia de que una infusión continua se relaciona con una menor incidencia de alteraciones gastrointestinales; sin embargo, esta incidencia diferirá de acuerdo con el parámetro de tolerancia gastrointestinal que sea considerado. Imagen elaborada por los autores.

parámetros de tolerancia GI. Considerando esto, se ha planteado como objetivo de investigación evaluar las diferencias reportadas entre la modalidad de infusión de nutrición enteral intermitente/bolos y la infusión continua en individuos en estado crítico.

\section{METODOLOGÍA}

\section{Estrategia de búsqueda y criterios de selección}

La estrategia de búsqueda utilizada a través de pregunta PICO (participants, exposure, comparison and outcomes) fue: ¿cuál es la diferencia entre infusión intermitente/ bolos y continua en la tolerancia gastrointestinal de pacientes en estado crítico que reciben nutrición enteral? Se realizó una revisión de la literatura para identificar literatura primaria que comparara las diferencias en la tolerancia GI entre ambas modalidades de infusión en el período de enero de 2010 a diciembre de 2020 . Se revisaron las bases de datos Pubmed, Proquest, Web of Science y Medline, utilizando los siguientes descriptores: intermittent feeding OR continuous feeding OR enteral tube feeding OR intermittent tube feeding OR continuous tube feeding OR intermittent feed OR continuous feed $O R$ intermittent enteral feeding $O R$ continuous enteral feeding OR enteral feeding OR continuous enteral nutrition OR intermittent enteral nutrition.

Se identificaron estudios observacionales (prospectivos y retrospectivos, cohortes, casos y controles) y ensayos clínicos aleatorizados realizados en pacientes en estado crítico. Fueron excluidos los estudios realizados en pacientes no críticos y revisiones narrativas de la literatura. Se seleccionaron aquellos estudios que reportaran información referente a la incidencia de síntomas GI en pacientes con NE en infusión intermitente/bolos y continua. 


\section{Extracción de datos}

Dos revisores capacitados (FGYE e IAOP) evaluaron de forma independiente los títulos y resúmenes de los estudios identificados para analizar posteriormente el texto en extenso de los estudios potencialmente relevantes. Una vez seleccionados los estudios conforme a los criterios de inclusión, se extrajeron las características de cada uno, tales como el nombre del primer autor, año de publicación, lugar de realización, diseño del estudio, edad y sexo de participantes, tamaño de la muestra, características de la intervención nutricional y resultados de tolerancia GI.

\section{RESULTADOS}

Se identificó un total de 27 estudios. Después de la verificación de criterios de inclusión, 9 fueron excluidos al tratarse de estudios de revisión, 2 al no describir claramente la intervención nutricional, 3 al no comparar la modalidad de infusión con NE intermitente o continua, 1 al no evaluar la tolerancia GI y 8 al tratarse de otro tipo de poblaciones (Figura 2). Finalmente, 4 estudios fueron incluidos en la revisión de la literatura, los cuales se muestran en la Tabla 1.

27 estudios identificados

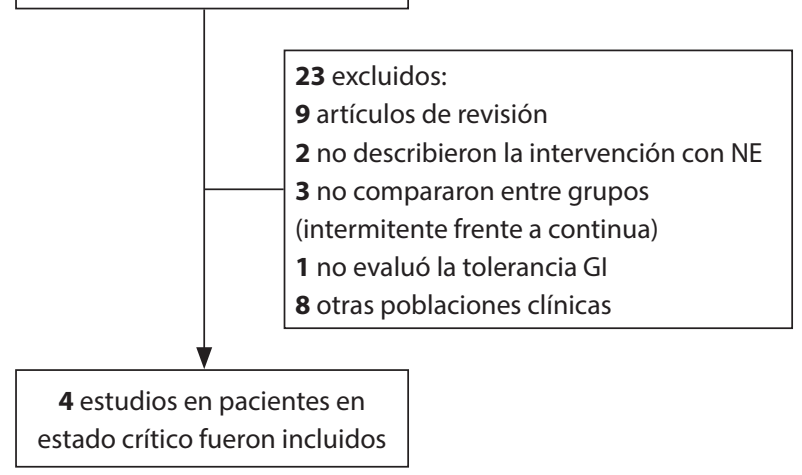

Figura 2. Diagrama de flujo de los artículos seleccionados. Elaborada por los autores.

\section{Complicaciones $\mathrm{Gl}$ asociadas con la modalidad de infusión de NE}

Maurya y colaboradores estudiaron las modalidades de alimentación en pacientes con traumatismo craneoencefálico que requirieron ventilación mecánica invasiva (VMI), el grupo con NE intermitente recibió 6 bolos cada 3 horas a través de una jeringa en un lapso de 18 horas; mientras que la modalidad continua fue infundida durante 18 horas a través de una bomba de alimentación. Los autores documentaron mayor residuo gástrico en el grupo con $\mathrm{NE}$ intermitente $(73 \pm$ 32,3 frente a 37,5 $\pm 32,4 \mathrm{~mL} ; p=0,002$ ), además de una mayor incidencia de episodios de diarrea (10\%), aunque sin diferencia estadística entre ambos grupos. Ninguna de las modalidades presentó casos de aspiración $^{(10)}$. En la Tabla 2 se menciona la prevalencia de intolerancias reportadas en los 4 estudios incluidos.

En pacientes críticos bajo VMI, Kadamani y colaboradores estudiaron el efecto de la utilización de infusión por bolos mediante gravedad a través de una jeringa (infusión en 10-15 minutos cada 4-6 horas), en comparación con la infusión continua (infundida durante 24 horas utilizando bomba de alimentación). Los autores reportaron una mayor incidencia de estreñimiento en el grupo tratado con infusión continua $(66,7 \%$ frente a $20 \%, p=0,025)$, además de una mayor incidencia de diarrea $(33,3 \%$ frente a $13,3 \%)$ y residuo gástrico $>200 \mathrm{~mL}(20 \%$ frente a $13,3 \%)$ en el grupo de infusión por bolos en comparación con el grupo en infusión continua, sin observar diferencias estadísticas entre las intervenciones ${ }^{(11)}$.

El estudio de Yağan y colaboradores evaluó la diferencia entre modalidades de infusión de NE en pacientes de la unidad de cuidados intensivos (UCI) con diagnóstico de disfagia, el primer grupo recibió $\mathrm{NE}$ mediante infusión intermitente (6 tomas de 30 minutos cada una) con $50 \mathrm{~mL}$ de fórmula e incrementos graduales de entre 50 y $100 \mathrm{~mL}$; mientras que el otro grupo recibió fórmula en infusión continua iniciando con $20 \mathrm{~mL} / \mathrm{h}$ y realizando incrementos de $20 \mathrm{~mL} / \mathrm{h}$ cada 6-8 horas. Ambas modalidades utilizaron bomba de alimentación y se observó una incidencia de vómito del $11,1 \%$ únicamente en el régimen intermitente. A diferencia de lo reportado previamente, los autores documentaron una mayor frecuencia de diarrea en el grupo de infusión continua (10,2\%) en comparación con la infusión intermitente $(5,5 \%)$. Aunque se evidenciaron estas complicaciones gastrointestinales, no se observaron diferencias significativas entre ambas modalidades de $\mathrm{NE}^{(12)}$.

En pacientes críticos con VMI, McNelly y colaboradores administraron una infusión intermitente de 6 tomas cada 4 horas mediante jeringa en un lapso de 3 a 5 minutos con un aporte inicial de $60-80 \mathrm{~mL}$ por bolo. Con respecto a la infusión continua, el volumen total fue administrado durante 24 horas. Los autores documentaron una prevalencia de diarrea para la infusión intermitente del $0 \%$ frente a 2,6 \% para la continua ( $p$ 
Tabla 1. Resultados primarios de los artículos incluidos

\begin{tabular}{|c|c|c|c|c|c|c|}
\hline Autor & País & $\begin{array}{l}\text { Diseño de } \\
\text { estudio }\end{array}$ & $\begin{array}{l}\text { Características } \\
\text { de la población }\end{array}$ & Población clínica & Intervención & Resultados primarios \\
\hline $\begin{array}{l}\text { Maurya I y } \\
\text { colaborado- } \\
\text { res, } 2011^{(10)}\end{array}$ & India & $\begin{array}{l}\text { Ensayo } \\
\text { clínico } \\
\text { aleatori- } \\
\text { zado }\end{array}$ & $\begin{array}{l}40 \text { pacientes: } \\
\text { - NE intermitente } \\
(n=20) \\
\text { - NE continua ( } n \\
=20)\end{array}$ & $\begin{array}{l}\text { - Pacientes en } \\
\text { estado crítico } \\
\text { con traumatismo } \\
\text { craneoencefálico } \\
\text { bajo ventilación } \\
\text { mecánica } \\
\text { - Edad: } 20 \text { a } 60 \\
\text { años }\end{array}$ & \begin{tabular}{|l} 
- \\
Infusión intermitente: \\
6 bolos cada $3 \mathrm{~h}$ \\
durante $18 \mathrm{~h}$ a través \\
de una jeringa \\
- Infusión continua: \\
para $18 \mathrm{~h} /$ día a través \\
de una bomba de \\
alimentación
\end{tabular} & $\begin{array}{l}\text { La medición del cociente } \\
\text { respiratorio, gasto } \\
\text { energético en reposo y } \\
\text { niveles de glucosa no fue } \\
\text { diferente entre los grupos. }\end{array}$ \\
\hline $\begin{array}{l}\text { Kadamani I y } \\
\text { colaborado- } \\
\text { res, } 2014^{(11)}\end{array}$ & Líbano & $\begin{array}{l}\text { Ensayo } \\
\text { clínico } \\
\text { pseudoa- } \\
\text { leatori- } \\
\text { zado }\end{array}$ & $\begin{array}{l}30 \text { pacientes: } \\
\text { - NE bolos ( } \mathrm{n} \\
=15 \text { ) } \\
\text { - NE continua ( } \\
=15)\end{array}$ & $\begin{array}{l}\text { - Pacientes en } \\
\text { estado crítico } \\
\text { con ventilación } \\
\text { mecánica } \\
\text { invasiva } \\
\text { - Edad: } 20 \text { a } 80 \\
\text { años }\end{array}$ & $\begin{array}{l}\text { - Infusión bolos: cada } \\
\text { 4-6 h por gravedad } \\
\text { a través de jeringa } \\
\text { durante } 10-15 \text { min } \\
\text { - Infusión continua: } \\
\text { para } 24 \text { h mediante } \\
\text { una bomba de } \\
\text { alimentación }\end{array}$ & $\begin{array}{l}\text { No se encontraron } \\
\text { diferencias en la } \\
\text { incidencia de aspiración. } \\
\text { Mayor incidencia de } \\
\text { estreñimiento con la } \\
\text { infusión continua. } \\
\text { No hubo diferencias en } \\
\text { otros síntomas Gl. }\end{array}$ \\
\hline $\begin{array}{l}\text { Yağan Ö y } \\
\text { colaborado- } \\
\text { res, 2017 }\end{array}$ & Turquía & $\begin{array}{l}\text { Ensayo } \\
\text { clínico } \\
\text { aleatori- } \\
\text { zado }\end{array}$ & $\begin{array}{l}37 \text { pacientes: } \\
\text { - NE intermitente } \\
(n=18) \\
\text { - NE continua ( } n \\
=19)\end{array}$ & $\begin{array}{l}\text { - Pacientes con } \\
\text { disfagia en la UCI } \\
\text { - Edad: } 18 \text { a } 85 \\
\text { años }\end{array}$ & \begin{tabular}{|l} 
- Infusión intermitente: \\
6 veces al día de 50 \\
$\mathrm{~mL}$ con incrementos \\
de $50-100 \mathrm{~mL}$, \\
infusión a través \\
de una bomba de \\
alimentación \\
- Infusión continua: \\
$20 \mathrm{~mL} / \mathrm{h}$ con \\
incrementos de 20 \\
$\mathrm{~mL}$ cada 6-8 h a \\
través de una bomba \\
de alimentación
\end{tabular} & $\begin{array}{l}\text { Los niveles de grelina y } \\
\text { leptina no presentaron } \\
\text { diferencia significativa } \\
\text { en ambos grupos de } \\
\text { alimentación. Solo se } \\
\text { observó un incremento } \\
\text { significativo en los niveles } \\
\text { de grelina en ambos } \\
\text { grupos a los } 7 \text { y } 14 \text { días, en } \\
\text { comparación con el primer } \\
\text { día }(p=0,003) \text {. }\end{array}$ \\
\hline $\begin{array}{l}\text { McNelly } \\
\text { AS y cola- } \\
\text { boradores, } \\
2020^{(13)}\end{array}$ & $\begin{array}{l}\text { Reino } \\
\text { Unido }\end{array}$ & $\begin{array}{l}\text { Ensayo } \\
\text { clínico } \\
\text { aleatori- } \\
\text { zado }\end{array}$ & \begin{tabular}{|l}
121 pacientes: \\
- \\
$\quad$ NE intermitente \\
- $n=62)$ \\
NE continua ( \\
$=59)$
\end{tabular} & $\begin{array}{l}\text { - Pacientes en } \\
\text { estado crítico } \\
\text { con ventilación } \\
\text { mecánica } \\
\text { invasiva } \\
\text { - } \\
\text { Edad: > } 18 \text { años }\end{array}$ & $\begin{array}{l}\text { - Infusión intermitente: } \\
6 \text { tomas cada } 4 \mathrm{~h} \\
\text { mediante jeringa } \\
\text { en un lapso de } 3 \text { a } 5 \\
\text { minutos } \\
\text { - Infusión continua: } \\
\text { volumen total } \\
\text { administrado para } \\
24 \mathrm{~h}\end{array}$ & $\begin{array}{l}\text { No se encontró diferencia } \\
\text { en la pérdida del músculo } \\
\text { recto femoral entre los } \\
\text { grupos a los } 10 \text { días. } \\
\text { Mayor aporte nutrimental } \\
\text { de energía y proteína } \\
\text { en el grupo con infusión } \\
\text { intermitente. } \\
\text { No hubo diferencias } \\
\text { en las concentraciones } \\
\text { plasmáticas de } \\
\text { aminoácidos entre los } \\
\text { grupos. }\end{array}$ \\
\hline
\end{tabular}

$=0,050)$, para vómito del $3,2 \%$ frente a $10,3 \%(p=$ $0,019)$ y para distensión abdominal del $3,2 \%$ frente a $0 \%$, respectivamente $(p=0,021)$. No se observaron diferencias significativas en la medición del residuo gástrico $>300 \mathrm{~mL}$ entre ambas modalidades de infusión $(19,7 \%$ frente a $15,4 \%)$. Los autores observaron una menor incidencia de interrupciones de la NE debido a náuseas y vómito, pero mayor por distensión abdominal en pacientes con infusión intermitente ${ }^{(13)}$.

\section{DISCUSIÓN}

$\mathrm{Al}$ momento de prescribir NE y elegir la modalidad de infusión de la fórmula, deberán tomarse en cuenta factores como la tolerancia esperada, características 
Tabla 2. Prevalencia de los síntomas gastrointestinales reportados

\begin{tabular}{|c|c|c|c|}
\hline Síntomas GI & $\begin{array}{l}\text { Pacientes, } \\
\text { n (\%) }\end{array}$ & $\begin{array}{c}\mathrm{NE} \\
\text { intermitente }\end{array}$ & $\begin{array}{c}\text { NE } \\
\text { continua }\end{array}$ \\
\hline $\begin{array}{l}\text { Maurya I y } \\
\text { colaboradores, } \\
2011^{(10)}\end{array}$ & $n=40$ & $n=20$ & $n=20$ \\
\hline - Residuo gástrico & & ND & ND \\
\hline - Vómito & & ND & ND \\
\hline $\begin{array}{l}\text { - Distensión } \\
\text { abdominal }\end{array}$ & & ND & ND \\
\hline - Estreñimiento & & ND & ND \\
\hline - Diarrea & & $2(10 \%)$ & 0 \\
\hline $\begin{array}{l}\text { Kadamani I y } \\
\text { colaboradores, } \\
2014^{(11)}\end{array}$ & $\mathbf{n}=\mathbf{3 0}$ & $n=15$ & $n=15$ \\
\hline $\begin{array}{l}\text { - Residuo gástrico } \\
\text { > } 200 \mathrm{~mL}\end{array}$ & & $3(20 \%)$ & $2(13,3 \%)$ \\
\hline - Vómito & & $1(6,7 \%)$ & $1(6,7 \%)$ \\
\hline $\begin{array}{l}\text { - Distensión } \\
\text { abdominal }\end{array}$ & & ND & ND \\
\hline - Estreñimiento & & $3(20 \%)$ & $10(66,7 \%)$ \\
\hline - Diarrea & & $5(33,3 \%)$ & $2(13,3 \%)$ \\
\hline $\begin{array}{l}\text { Yağan Ö y } \\
\text { colaboradores, } \\
2017^{(12)}\end{array}$ & $n=37$ & $n=18$ & $n=19$ \\
\hline - Residuo gástrico & & ND & ND \\
\hline - Vómito & & $2(11,1 \%)$ & $0 \%$ \\
\hline $\begin{array}{l}\text { - Distensión } \\
\text { abdominal }\end{array}$ & & ND & ND \\
\hline - Estreñimiento & & ND & ND \\
\hline - Diarrea & & $1(5,5 \%)$ & $2(10,2 \%)$ \\
\hline $\begin{array}{l}\text { McNelly AS y } \\
\text { colaboradores, } \\
2020^{(13)}\end{array}$ & $n=121$ & $n=62$ & $n=59$ \\
\hline $\begin{array}{l}\text { - Residuo gástrico } \\
\text { > } 300 \mathrm{~mL}\end{array}$ & & $31(19,7 \%)$ & $24(15,4 \%)$ \\
\hline - Vómito & & $5(3,2 \%)$ & $16(10,3 \%)$ \\
\hline $\begin{array}{l}\text { - Distensión } \\
\text { abdominal }\end{array}$ & & $5(3,2 \%)$ & $0 \%$ \\
\hline - Estreñimiento & & ND & ND \\
\hline - Diarrea & & $0 \%$ & $4(2,6 \%)$ \\
\hline
\end{tabular}

Gl: gastrointestinales; ND: no determinado; NE: nutrición enteral. de la fórmula, sitio de administración (gástrica o en el intestino delgado), requerimientos nutricionales, disponibilidad de las fórmulas enterales e insumos para su administración ${ }^{(14)}$.

Las principales complicaciones que pueden ocurrir durante la infusión de NE son náuseas, vómito, retraso en el vaciamiento gástrico, diarrea, estreñimiento y dolor abdominal tipo cólico ${ }^{(15)}$. Se ha observado que la intolerancia a la $\mathrm{NE}$ en los pacientes en estado crítico tratados con VMI puede ocurrir hasta en el $30 \%$ de los casos, de los cuales en un 61,6 \% se observó incremento en el residuo gástrico, vómito/diarrea en un $36,6 \%$ y distensión abdominal en el 16,6 \%. A su vez, estas intolerancias se relacionan con menor adecuación nutrimental, menor cantidad de días libres de ventilador, estancia prolongada en la UCI e incremento en la mortalidad $^{(6,16)}$.

El impacto de las modalidades de infusión de NE (intermitente/bolos frente a continua) en la incidencia de intolerancia GI ha cobrado relevancia en los últimos años; sin embargo, la mayoría de la evidencia actual ha diferido sobre la definición de estas modalidades y no ha abordado como resultado primario la tolerancia GI.

Un metaanálisis realizado en población en estado crítico publicado por $\mathrm{Ma} \mathrm{Y}$ y colaboradores reportó que la incidencia de diarrea, vómito y distensión abdominal es similar entre ambas modalidades de alimentación, y se registró un incremento en el volumen de residuo gástrico (RR: 3,62; IC $95 \%$ : 1,43-9,12, $p=0,006$ ) y mayor riesgo de aspiración (RR: 3,29; IC $95 \%$ : 1,189,16; $p=0,02$ ) luego del uso de infusión intermitente durante más de una semana. Otros resultados como menor estreñimiento (RR: 0,66; IC $95 \%$ : 0,45-0,98; $p=0,04$ ) y mayor aporte calórico (desviación media [DM]: 184,81; IC $95 \%: 56,61-313,01, p=0,005)$ fueron observados a favor del régimen intermitente. Los autores concluyen que la infusión continua se asocia con menor intolerancia a la NE, tomando en cuenta el incremento en el volumen de residuo gástrico y aspiración $^{(17)}$. En su metodología, consideran que la intolerancia a la NE corresponde a episodios en los que se interrumpe la infusión debido a diarrea, estreñimiento, vómito y distensión abdominal, o si no se ha logrado cubrir el $80 \%$ de los requerimientos en las primeras 72 horas. Los puntos de corte definidos para cada episodio de intolerancia varían entre un estudio y otro, por lo que resulta complicado poder hacer comparativos entre ambas modalidades de infusión.

La mayoría de los ensayos clínicos ha tenido como objetivo evaluar el impacto del modo de infusión de la 
NE en otros desenlaces, y se ha documentado una posible disminución en el riesgo de mortalidad en pacientes críticos con ventilación mecánica y traumatismo, sin documentar diferencias en la incidencia de neumonía, infecciones, estancia hospitalaria o adecuación nutricional $^{(18)}$. Es necesario que los ensayos clínicos evalúen estos parámetros de tolerancia GI como objetivo principal, ya que en los estudios presentados en esta revisión no fue el caso.

\section{CONCLUSIONES}

La incidencia de intolerancia GI entre la infusión intermitente/bolos y continua es muy variable, debido a heterogeneidad de definiciones. No existen ensayos clínicos aleatorizados cuyo objetivo principal haya sido evaluar la incidencia de intolerancia GI luego de comparar las modalidades de infusión. Existe evidencia a favor de la utilización de una infusión continua sobre una menor incidencia de intolerancia a la NE; sin embargo, esto varía de acuerdo con qué parámetro de tolerancia GI se tenga en cuenta.

\section{Fuente de financiación}

Ninguna.

\section{Conflicto de intereses}

Los autores no declaran ningún conflicto de intereses.

\section{Contribución de autores}

IAOP y FGYE contribuyeron con el diseño de la investigación, análisis e interpretación de los datos. NCRM participó en la escritura del artículo y en la edición del mismo. Los autores redactaron, revisaron y aprobaron el manuscrito en su forma final.

\section{Referencias bibliográficas}

1. McClave SA, Omer E. Clinical nutrition for the gastroenterologist: the physiologic rationale for providing early nutritional therapy to the hospitalized patient. Curr Opin Gastroenterol. 2020;36(2):118-121. doi: 10.1097/ MOG.0000000000000618

2. Quiroz-Olguín G, Gutiérrez-Salmeán G, Posadas-Calleja JG, Padilla-Rubio MF, Serralde-Zúñiga AE. The effect of enteral stimulation on the immune response of the intestinal mucosa and its application in nutritional support. Eur J Clin Nutr. 2021. doi: 10.1038/s41430-021-00877-7

3. Pletschette Z, Preiser JC. Continuous versus intermittent feeding of the critically ill: have we made progress?
Curr Opin Crit Care. 2020;26(4):341-345. doi: 10.1097/ MCC. 0000000000000733

4. Puthucheary Z, Gunst J. Are periods of feeding and fasting protective during critical illness? Curr Opin Clin Nutr Metab Care. 2021;24(2):183-188. doi: 10.1097/ MCO.0000000000000718

5. Heyland DK, Ortiz A, Stoppe C, Patel JJ, Yeh DD, Dukes G, et al. Incidence, Risk Factors, and Clinical Consequence of Enteral Feeding Intolerance in the Mechanically Ventilated Critically Ill: An Analysis of a Multicenter, Multiyear Database. Crit Care Med. 2021;49(1):49-59. doi: 10.1097/ CCM.0000000000004712

6. Reintam Blaser A, Preiser JC, Fruhwald S, Wilmer A, Wernerman J, Benstoem C, et al. Gastrointestinal dysfunction in the critically ill: a systematic scoping review and research agenda proposed by the Section of Metabolism, Endocrinology and Nutrition of the European Society of Intensive Care Medicine. Crit Care. 2020;24(1):224. doi: 10.1186/s13054-020-02889-4

7. Singer P, Blaser AR, Berger MM, Alhazzani W, Calder PC, Casaer MP, et al. ESPEN guideline on clinical nutrition in the intensive care unit. Clin Nutr. 2019;38(1):48-79. doi: 10.1016/j.clnu.2018.08.037

8. McClave SA, Taylor BE, Martindale RG, Warren MM, Johnson DR, Braunschweig C, et al. Guidelines for the Provision and Assessment of Nutrition Support Therapy in the Adult Critically Ill Patient: Society of Critical Care Medicine (SCCM) and American Society for Parenteral and Enteral Nutrition (A.S.P.E.N.). JPEN J Parenter Enteral Nutr. 2016;40(2):159-211. doi: 10.1177/0148607115621863

9. Elke G, Hartl WH, Kreymann KG, Adolph M, Felbinger TW, Graf T, et al. Clinical Nutrition in Critical Care Medicine Guideline of the German Society for Nutritional Medicine (DGEM). Clin Nutr ESPEN. 2019;33:220-275. doi: 10.1016/j.clnesp.2019.05.002

10. Maurya I, Pawar M, Garg R, Kaur M, Sood R. Comparison of respiratory quotient and resting energy expenditure in two regimens of enteral feeding - continuous vs. intermittent in head-injured critically ill patients. Saudi J Anaesth. 2011;5(2):195-201. doi: 10.4103/1658-354X.82800

11. Kadamani I, Itani M, Zahran E, Taha N. Incidence of aspiration and gastrointestinal complications in critically ill patients using continuous versus bolus infusion of enteral nutrition: a pseudo-randomised controlled trial. Aust Crit Care. 2014;27(4):188-93. doi: 10.1016/j.aucc.2013.12.001

12. Yağan Ö, Taş N, Ayyildiz SN, Karakahya M, Noyan T. Comparison of the effects of continuous versus intermittent enteral feeding on plasma leptin and ghrelin levels in Intensive Care Units. Rev Nutr. 2017;30(4):409-418. doi:10.1590/1678-98652017000400001

13. McNelly AS, Bear DE, Connolly BA, Arbane G, Allum L, Tarbhai A, et al. Effect of Intermittent or Continuous Feed on Muscle Wasting in Critical Illness: A Phase 2 
Clinical Trial. Chest. 2020;158(1):183-194. doi: 10.1016/j. chest.2020.03.045

14. Patel JJ, Rosenthal MD, Heyland DK. Intermittent versus continuous feeding in critically ill adults. Curr Opin Clin Nutr Metab Care. 2018;21(2):116-120. doi: 10.1097/ MCO.0000000000000447

15. Btaiche IF, Chan LN, Pleva M, Kraft MD. Critical illness, gastrointestinal complications, and medication therapy during enteral feeding in critically ill adult patients. Nutr Clin Pract. 2010;25(1):32-49. doi: 10.1177/0884533609357565

16. Gungabissoon U, Hacquoil K, Bains C, Irizarry M, Dukes G, Williamson R, et al. Prevalence, risk factors, clinical consequences, and treatment of enteral feed intolerance during cri- tical illness. JPEN J Parenter Enteral Nutr. 2015;39(4):441-8. doi: $10.1177 / 0148607114526450$

17. Ma Y, Cheng J, Liu L, Chen K, Fang Y, Wang G, Zhu J, Chen L. Intermittent versus continuous enteral nutrition on feeding intolerance in critically ill adults: A meta-analysis of randomized controlled trials. Int J Nurs Stud. 2021;113:103783. doi: 10.1016/j.ijnurstu.2020.103783

18. Heyland DK, Patel J, Hill A, Lee Z-Y, Yap C, Ortiz LA, et al. Enteral Nutrition (Other): Continuous vs. Other Methods of Administration [internet]. Critical Care Nutrition; 2021 [consultado el 22 de septiembre de 2021]. Disponible en: https://www.criticalcarenutrition.com/docs/6.3\%20 Continuous_2April\%205,\%202021.pdf 